\title{
Site-Specific Excitation in Free Krypton Clusters
}

\author{
A. Knop and B. Wassermann \\ Fachbereich Chemie, Freie Universität Berlin, Takustrasse 3, D-14195 Berlin, Germany \\ E. Rühl \\ Fachbereich Physik, Universität Osnabrück, Barbarastrasse 7, D-49069 Osnabrück, Germany
}

(Received 29 September 1997)

\begin{abstract}
Site-specific excitation in $\mathrm{Kr}$ clusters is investigated by high resolution inner-shell excitation in combination with model calculations, which are based on a core exciton model. Partial cation and total electron yield spectra of variable size $\mathrm{Kr}$ clusters are reported for the $\mathrm{Kr} 3 d$ excitation regime (90-96 eV) using synchrotron radiation. A cluster size-dependent spectral evolution is observed, corresponding to the transition from low lying cluster Rydberg states into surface and bulk exciton states. The results indicate that individual sites in perfectly and imperfectly shaped clusters are clearly distinguished. [S0031-9007(98)05596-3]
\end{abstract}

PACS numbers: $36.40 . \mathrm{Mr}, 61.10 . \mathrm{Ht}$

Clusters play a central role in linking properties of isolated atoms to those of the condensed phase of matter [1,2]. The size-dependent evolution of the electronic and geometric structure is therefore of primary interest. Van der Waals clusters, such as rare gas clusters, are ideal systems to study size effects in a systematic way. Rare gas clusters are known to consist of geometrical structures which are not well defined since perfect icosahedral structures [3] and imperfect structures [4] may be generated in jet expansions, a typical cluster production technique. Resonant photon excitation of clusters is a sensitive method to probe changes in electronic structure as a function of cluster size. Recent instrumental developments in the field high resolution $\mathrm{x}$-ray spectroscopy using synchrotron radiation make high spectral resolution experiments on free clusters in the gas phase possible $[4,5]$. The inherent advantage in exciting highly localized core levels is due to element specific, and often site specific, processes which cannot be obtained by valence shell excitations.

In this Letter we present experimental results supported by model calculations. High spectral resolution in combination with results from model calculations gives, to our knowledge for the first time, site-specific information on absorption processes of homogeneous clusters occurring in the inner-shell excitation regime. We have chosen the $\mathrm{Kr} 3 d$ regime $(90-100 \mathrm{eV})$, since it is well investigated for the atom [6] and the solid [7]. The preedge regime is dominated by two series of Rydberg states $\left(\mathrm{Kr} 3 d_{5 / 2} \rightarrow n p\right.$ and $\left.\mathrm{Kr} 3 d_{3 / 2} \rightarrow n p\right)$ in the atom, whereas in the solid the broad features of low lying core excitons are observed.

The experimental setup consists of a jet expansion [8]. The skimmed jet is crossed with the beam of monochromatic synchrotron radiation (BESSY-I, beam line: U2FSGM [9]). Cations are accelerated into a time-of-flight mass spectrometer. Total electron yields (TEY) are extracted into the opposite side using a channeltron adjacent to the cluster beam. Yields of mass-selected cluster cations are used for experiments on electronic properties of small clusters (typical size range: $\mathrm{Kr}_{n}$, with $n<70$ ). Fragmentation is initiated in the $\mathrm{Kr} 3 d$ regime by resonant MNNAuger decays of the core hole leading predominantly to doubly charged clusters [10], which are known to decay via Coulomb explosion [11]. Properties of large clusters are investigated by TEY spectra. The average cluster size $\langle N\rangle$ of neutral clusters is estimated according to correlations of the reduced scaling parameter $\Gamma^{*}$ with $\langle N\rangle[12,13]$. We have taken the $\Gamma^{*}-\langle N\rangle$ calibration derived by Karnbach et al. [13], which gives more realistic values than the earlier results of Farges et al. [14]. TEY spectra of solid multilayers are obtained using an ultrahigh vacuum setup.

Spectral shifts of resonant excitations in clusters relative to the atomic Rydberg states are modeled by using the concept of core exciton shifts in clusters. The radial Schrödinger equation is used in the frame of the nonstructural core exciton theory [15]. The potential for $r<R_{m}$ is taken to be $U_{n}=-C_{n}$, where $r$ is the electron hole-pair distance, $R_{m}$ is the atomic radius, $C_{n}$ is a constant, and $n$ is the principal quantum number of the Rydberg level. The constants $C_{n}$ must be such that the energy values of the atomic Schrödinger equation give the exact experimental atomic excitation energies [15]. The screened potential $U_{n}=1 / \epsilon r$ term is substituted in the interstitial region $\left(r>R_{m}\right)$ by the sum of the Rydberg term $U_{n}=1 / r$ and the perturbing electron-neutral atom pseudopotential [16],

$$
\begin{aligned}
V_{e^{-}-\mathrm{Kr}}=\sum_{i} V_{n}^{i}= & \sum_{i}\left[\frac{A}{\left(B+\rho_{i}^{6}\right) \rho_{i}^{4}}-\frac{\left[\vec{\rho}_{i} \vec{\rho}_{i}\right] S\left(\rho_{i}\right)}{2 \rho_{i}^{3}}\right] \\
& \times \exp \left[-\left(\frac{a}{n_{\mathrm{ref}} \rho_{i}}\right)^{4}\right]
\end{aligned}
$$

augmented by a suitable trial cutoff function in the form of an $S$-wave solution of the radial Schrödinger equation accounting for the interaction of a single electron with a $\mathrm{Kr}$ atom. $\vec{\rho}_{i}=\left|\vec{r}-\vec{R}_{i}\right|$ is the electron- $i$ th-neutral atom distance, $a$ is the electron scattering length in the single atom 
limit, and $n_{\text {ref }}$ is the refractive index. The value of the electron scattering length $a$ is obtained from the elastic scattering cross section as a first approximation. It is further optimized for the spectral shift of the dimer with $n_{\text {ref }}=1$ and is kept constant for all other cluster sizes. The attenuation of the electron scattering length through the refractive index approximates the refractive properties of the cluster environment, caused by multiple reflections of the electron wave from the surrounding atoms. The constants $A$ and $B$ have the following meaning [17]: $A$ is the polarizability multiplied by a constant (const) and $B$ is also a constant which is related to $r_{0}$, with $r_{0}=(\text { const }-B)^{1 / 6}$. Here, $r_{0}$ is the distance where the potential goes first to zero. The competition between screening of the electron, due to repulsive scattering, and the increased electron binding, due to cluster polarization, determines the sign of the overall spectral shift relative to the atomic transition. $a$ and $n_{\text {ref }}$ are not fit parameters, since they have a physical meaning. In the model, $n_{\text {ref }}$ ranges between 1.0 (dimer), $1.28\left(\mathrm{Kr}_{13}\right)$, and 1.336 (bulk of $\mathrm{Kr}_{146}$ ). The perturbation is approximated by the sum of the atomic potentials $V_{i}$, whereas the long-range cluster size- and structure-dependent contributions are considered by the many-body polarization energy factor. Here, $\vec{p}_{i}=\alpha \vec{E}_{i}$ is the dipole moment at the atom $i$ induced by the electron-hole pair, $\alpha$ is the atomic polarizability, and $\vec{E}_{i}$ is the total electric field at the location of the atom $i$. The latter quantity is calculated iteratively, using the procedure outlined earlier [18]. The switching function $S\left(\rho_{i}\right)$ [19] is designed to gradually turn off the effective electron charge inside the atomic core. We use the angular average of the radial potential, where the first order energy correction is expressed by [19]

TABLE I. Calculated shifts of the $\mathrm{Kr} 3 d_{5 / 2} \rightarrow 5 p$ transition in $\mathrm{Kr}$ clusters relative to the atomic transition $(91.200 \mathrm{eV})$.

\begin{tabular}{llr}
\hline \hline \multicolumn{1}{c}{ Cluster } & \multicolumn{1}{c}{ Site } & Shift [meV] \\
\hline $\mathrm{Kr}_{2}$ (icosahedron) & corner & -28 \\
$\mathrm{Kr}_{13}$ (icosahedron) & center (bulk) & 96 \\
& corner & 305 \\
$\mathrm{Kr}_{55}$ (icnter (bulk) & 124 \\
& edge & 443 \\
$\mathrm{Kr}_{147}$ (icosahedron) & corner & 185 \\
& center (bulk) & 136 \\
& edge & 441 \\
& face & 182 \\
& second shell & 205 \\
$\mathrm{Kr}_{44}$ (octahedron) & corner & 287 \\
& center (bulk) & 71 \\
$\mathrm{Kr}_{146}$ (octahedron) & edge & 381 \\
& corner & 132 \\
& center (bulk) & 89 \\
& edge & 384 \\
$\mathrm{Kr}_{146}$ (defect oct.) & face & 141 \\
$\mathrm{Kr}_{22}$ (hcp embryo) & edge (stacking fault) & 185 \\
& surface & 141 \\
& center (bulk) & 106 \\
\hline \hline
\end{tabular}

$$
\begin{aligned}
\Delta E_{n}^{1}=\sum_{i}\left\langle\chi_{n}\left|V_{n}^{i}\right| \chi_{n}\right\rangle= & \sum_{i} \int_{0}^{\infty} d r\left|\chi_{n}\left(r, R_{i}\right)\right|^{2} \\
& \times \int \frac{d \Omega}{4 \pi} V_{n}^{i}\left(r, R_{i}, \Omega\right) .
\end{aligned}
$$

The logarithmic derivative of the envelope wave function $\chi_{n}$ is matched for $r=R_{m}$ [20] including proper normalization of the wave function. Calculations are performed by investigating excitations of equivalent sites of a cluster in order to control the accuracy of the integration procedure. These are found to differ on the order of $1 \%-2 \%$. Several perfect and imperfect cluster structures are considered. Icosahedral shapes are known to represent the most stable configurations of small rare gas clusters [3]. Therefore, perfectly shaped icosahedra, cuboctahedra, as well as imperfect species, i.e., structures with stacking faults, as well as a hypothetical cluster $\mathrm{Kr}_{22}$, which has been proposed to represent the embryo for fast fcc-cluster growth [21], are considered (cf. Table I).

Figure 1 compares a series of spectra showing the spectral evolution as a function of $\langle N\rangle$ in the $\mathrm{Kr} 3 d$ excitation regime $(90-96 \mathrm{eV})$. The atomic spectrum is represented by the $\mathrm{Kr}^{+}$yield [Fig. 1(a)]. The spectra $1(b)-1(g)$ correspond to an increase in $\langle N\rangle$, ranging from $\langle N\rangle=2$ to $\langle N\rangle=1250 . \mathrm{Kr}_{2}{ }^{+}$yields are used for investigating properties of small clusters. Properties

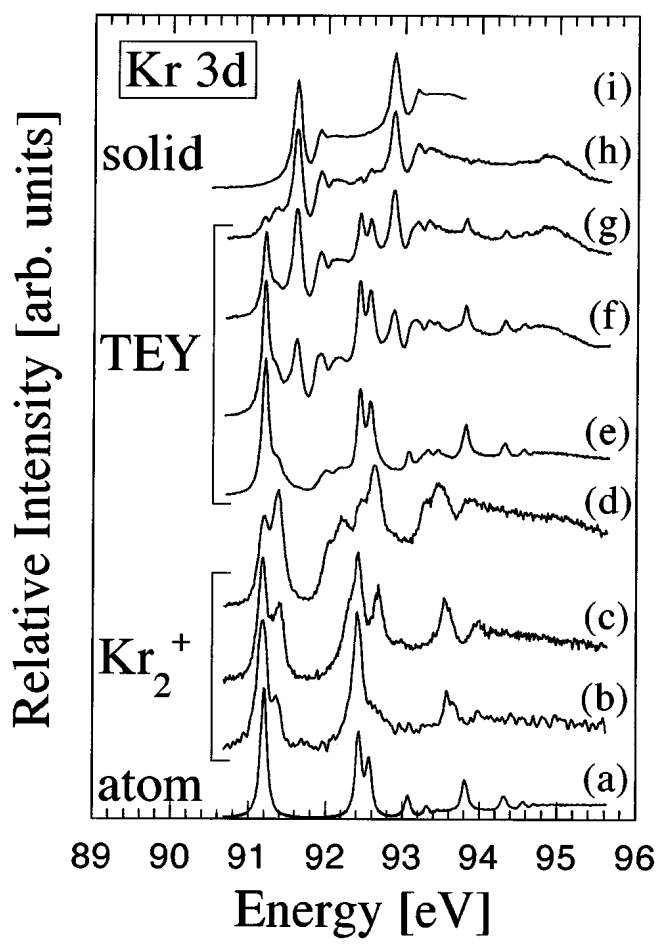

FIG. 1. Series of spectra of $\mathrm{Kr}$ clusters recorded in the $\mathrm{Kr} 3 d$ excitation regime. $(b)-(d) \mathrm{Kr}_{2}{ }^{+}$yields corresponding to $\langle N\rangle=2,12$, and 30 , respectively. $(e)-(g)$ Total electron yields (TEY) corresponding to $\langle N\rangle=90,350$, and 1250, respectively. (h) Same as $(g)$, where $15 \%$ atomic signal is subtracted. The atomic spectrum $\left[\mathrm{Kr}^{+}\right.$yield $\left.1(a)\right]$ and a TEY of the solid $(i)$ are included. 
of large clusters are studied by TEY spectra [cf. Fig. 1: $(e)-(g)]$. These show visible components of the atomic spectrum, even in the high $\langle N\rangle$ regime [cf. Fig. $1(g)$ ]. The atomic component is visible since it is not suppressed as in $\mathrm{Kr}_{2}{ }^{+}$spectra. In addition, the absorption cross section of the low lying atomic Rydberg states is about 3 times higher compared to the corresponding core exciton states of the solid [7]. Figure $1(h)$ is obtained from Fig. $1(g)$, where a portion of $15 \%$ atomic contribution is subtracted. This contribution is due to electrons which come from the atomic surroundings of the cluster beam. As a result, spectrum $1(h)$ is similar in shape to that of the solid [Fig. 1(i)]. All transitions are split by $1.2 \mathrm{eV}$ as a result of spin-orbit splitting [7]. The resonances shown in Fig. 1 change their energy positions as a function of $\langle N\rangle$ in a systematic way. All features below $91.6 \mathrm{eV}$ are due to $\mathrm{Kr} 3 d_{5 / 2} \rightarrow 5 p$ transitions. The corresponding spin-orbit components $\left(\mathrm{Kr} 3 d_{3 / 2} \rightarrow 5 p\right)$ are found between 92.3 and $92.8 \mathrm{eV}$. Broad features in the regimes $91.8-92.3 \mathrm{eV}$ and $93.0-93.5 \mathrm{eV}$ are due to higher lying $\mathrm{Kr} 3 d \rightarrow n p$ surface and bulk excitations. They show, as a general trend, a redshift relative to the atomic transitions as a function of $\langle N\rangle$, which is directly related to the $\mathrm{Kr} 3 d$ ionization energies which

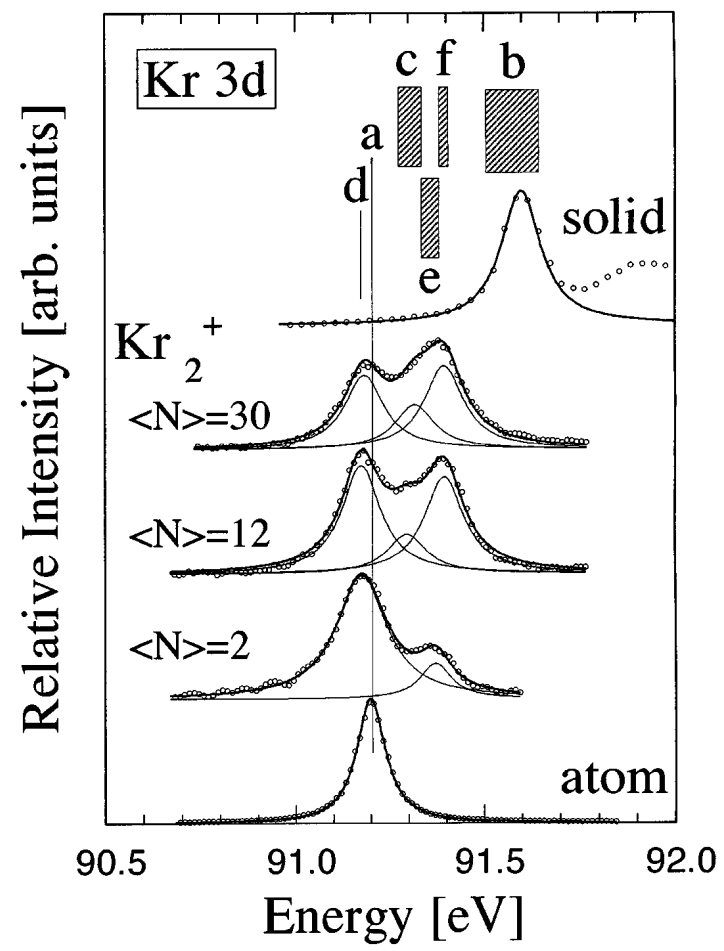

FIG. 2. Photoion yields of mass selected $\mathrm{Kr}_{2}{ }^{+}$near the $\mathrm{Kr}_{3} d_{5 / 2} \rightarrow 5 p$ transition at various average cluster sizes $\langle N\rangle$ (open circles). The $\mathrm{Kr}^{+}$yield (bottom) and the TEY of the solid (top) are included. The deconvolution of the experimental cluster spectra is performed using Lorentzians (solid lines; linewidth: $125 \mathrm{meV}$ ). A comparison is made with the calculated core exciton shifts in clusters: $a$ : atom; $b$ : bulk; $c$ : corner; $d$ : dimer, $e$ : edge; and $f$ : face. The shaded areas correspond to value ranges which are due to different geometries and cluster sizes (cf. Table I). drop by about $1 \mathrm{eV}$ from the atom to the bulk [22]. In addition, the linewidths $\Gamma$ are greater in cluster and condensed phase spectra $(\Gamma=125 \mathrm{meV})$ compared to the atomic spectrum $[\Gamma=95 \mathrm{meV}$ (cf. [23])]. The width of the atomic transitions is due to the core hole lifetime. Increased linewidths in the pre-edge regime of clusters are due to repulsive final states leading to fast decay processes [24], so that some of the lines are blended. Lorentzian line shapes are appropriate to fit the experimental features. We use the same line shape for all cluster sizes since only the $\mathrm{Kr} 3 d_{5 / 2} \rightarrow 5 p$ transition is investigated. Further information on spectral assignments as a function of $\langle N\rangle$ is therefore derived from spectral deconvolutions, as shown in Figs. 2 and 3, including results of our model calculations. Energy ranges corresponding to $\mathrm{Kr} 3 d_{5 / 2} \rightarrow$ $5 p$ transitions of individual sites are marked at the top of both figures (cf. Table I). In general, sites in small clusters represent the lower energy part of the marked ranges. Edge and bulk sites absorb at higher energy in icosahedra compared to cuboctahedra. This gives, in combination with the experimental results, site-specific information on the absorption of individual atoms in clusters. The $\mathrm{Kr}_{2}{ }^{+}$yield, corresponding to $\langle N\rangle=2$, is similar to that of the atom, with a slight redshift of $25 \mathrm{meV}$ of the discrete resonances relative to the

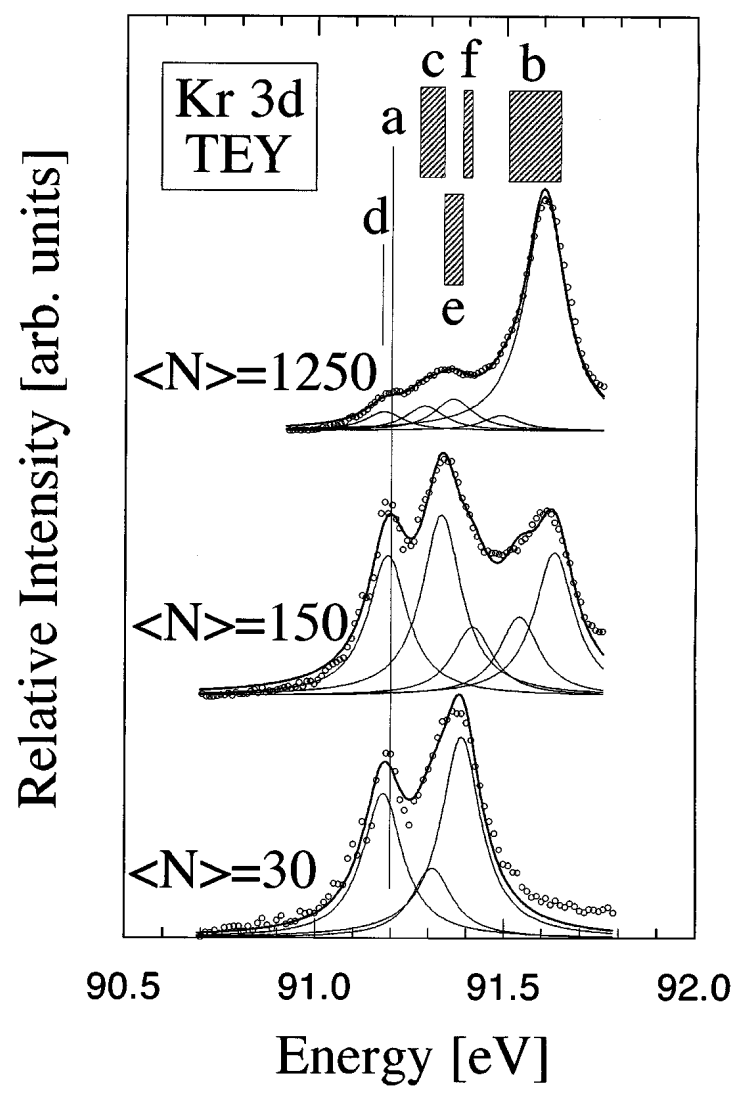

FIG. 3. Total electron yield (TEY) spectra near the $\mathrm{Kr} 3 d_{5 / 2} \rightarrow 5 p$ transition at various average cluster sizes $\langle N\rangle$. A contribution of the atomic signal is subtracted. For further details, see Fig. 2. 
atomic values $\left(\mathrm{Kr} 3 d_{5 / 2} \rightarrow 5 p: 91.200 \mathrm{eV} ; \mathrm{Kr} 3 d_{3 / 2} \rightarrow\right.$ $5 p: 92.425 \mathrm{eV}$ [6]). The model calculations indicate that the dimer $\mathrm{Kr} 3 d \rightarrow 5 p$ absorptions are redshifted by $28 \mathrm{meV}$, which agrees with the experimental findings. The blueshifted shoulder at $91.367 \mathrm{eV}$ is represented by a single Lorentzian line. Since only small clusters are present in the jet at $\langle N\rangle=2$, we assign this feature to the absorption of clusters which are only slightly larger than dimers. The $\mathrm{Kr}_{2}{ }^{+}$yields, corresponding to $\langle N\rangle=$ 12 and 30, show clear evidence that three components are required to fit the experimental spectra. A weak component is less blueshifted than the other stronger one. The weak component is assigned to corner sites in clusters of icosahedral shape and surface sites of imperfectly shaped clusters, such as $\mathrm{Kr}_{22}$, whereas edge sites coming from fragmentation of larger clusters represent the high energy component. The $\mathrm{Kr}_{2}{ }^{+}$signal reflects, therefore, fragmentation of larger clusters which consist of only a small fraction to corner sites and more abundantly to edge sites. Face and bulk sites are expected to be present only in clusters which contain considerable amounts of bulk atoms. These are evidently not observed in the series of $\mathrm{Kr}_{2}{ }^{+}$spectra (cf. Fig. 2). We have subtracted a variable fraction of the atomic component from the TEY spectra in order to obtain electronic properties of large clusters. The results are presented in Fig. 3 along with spectral fits. The bottom spectrum of Fig. $3(\langle N\rangle=30)$ gives a direct comparison to the corresponding $\mathrm{Kr}_{2}{ }^{+}$yield shown in Fig. 2, indicating that both spectra are almost identical. This is a clear indication that both detection methods give similar results in a size regime that applies to both methods. The TEY corresponding to $\langle N\rangle=150$ shows the strongest component for edge sites in cuboctahedrally shaped clusters. A similar absorption energy corresponds to corner sites in icosahedra, but these are expected to be weak at $\langle N\rangle=150$. In addition, stacking faults in defect octahedra will enhance the intensity of this absorption. These findings point to the fact that fast growing clusters reaching large sizes are imperfectly shaped with fccstructural elements [4,21]. There is also evidence for face sites as well as two different bulk components, corresponding to subsurface layers and the bulk interior of the clusters. The critical size range for the occurrence of bulk excitons is found to be around $\langle N\rangle=90$. This is unexpected since, for the perfect icosahedron $\mathrm{Kr}_{55}$, one would expect that $23 \%$ of the atoms are already located in the bulk [3]. It is another piece of evidence that imperfect cluster structures are formed with increasing $\langle N\rangle$, which have a higher surface-to-bulk ratio than the corresponding perfectly shaped icosahedra. The TEY at $\langle N\rangle=1250$ is dominated by the bulk exciton component. All other sites are considerably weaker in intensity. The strong bulk exciton signal is not likely to reflect deviations from the determination of $\langle N\rangle$ rather than an enhanced sensitivity of the TEY signal to low kinetic energy electrons which are formed by inelastic scattering in the bulk of the clusters. This is in agreement with previous experiments on zero kinetic energy photoelectron spectroscopy of core-excited argon clusters [25].

In conclusion, we have shown that high resolution spectroscopy on $3 d$-excited $\mathrm{Kr}$ clusters gives in combination with model calculations, for the first time, site-specific information on inner-shell absorption processes in homogeneous $\mathrm{Kr}$ clusters. Experimental evidence for these findings comes from site- and size-dependent shifts of resonant absorption lines in the $\mathrm{Kr} 3 d$ pre-edge regime. A comparison of our model calculations to the experimental data shows that imperfect structures dominate the spectra, especially in the large cluster regime.

We thank G. Reichardt (BESSY) for his help in running the U2-FSGM, C.M. Teodorescu, D. Gravel, and I. T. Steinberger for their help with the spectra of the solid. Financial support by the Bundesministerium für Bildung, Wissenschaft, Forschung und Technologie (BMBF), the Fonds der Chemischen Industrie, and the Deutsche Forschungsgemeinschaft are gratefully acknowledged.

[1] J. Jortner, Ber. Bunsenges. Phys. Chem. 88, 188 (1984).

[2] Clusters of Atoms and Molecules, edited by H. Haberland (Springer, Berlin, 1994), Vol. I; ibid. (Springer, Berlin, 1995), Vol. II.

[3] M. R. Hoare, Adv. Chem. Phys. 40, 49 (1979).

[4] S. Kakar et al., Phys. Rev. Lett. 78, 1675 (1997).

[5] F. Federmann et al., Phys. Rev. Lett. 73, 1549 (1994); O. Björneholm et al., Phys. Rev. Lett. 74, 3017 (1995).

[6] G. C. King et al., J. Phys. B 10, 2479 (1977).

[7] R. Haensel et al., Phys. Rev. B 7, 1577 (1973).

[8] E. Rühl et al., J. Chem. Phys. 98, 2653 (1993).

[9] J. Bahrdt et al., Rev. Sci. Instrum. 63, 339 (1992).

[10] N. Saito and I. H. Suzuki, in Proceedings of the VUV-10 Conference, MO-81, Paris 1992 (to be published).

[11] O. Echt et al., Phys. Rev. A 38, 3236 (1988).

[12] O. F. Hagena, Z. Phys. D 4, 291 (1987).

[13] R. Karnbach et al., Rev. Sci. Instrum. 64, 2838 (1993).

[14] J. Farges et al., J. Chem. Phys. 84, 3491 (1986).

[15] L. Resca et al., Phys. Rev. B 18, 696 (1978); L. Resca et al., Phys. Rev. B 18, 702 (1978).

[16] G. Martyna and B. J. Berne, J. Chem. Phys. 90, 3744 (1989).

[17] D. F. Coker et al., J. Chem. Phys. 86, 5689 (1987).

[18] O. Björneholm et al., J. Chem. Phys. 104, 1846 (1996).

[19] C. Cohen-Tannoudji et al., Quantum Mechanics (Wiley, New York, 1972), Vol. II, p. 1142.

[20] I. V. Abarenkov and V. Heine, Philos. Mag. 12, 529 (1965).

[21] B. W. van de Waal, Z. Phys. D 20, 349 (1991); J. Chem. Phys. 98, 4909 (1993); Phys. Rev. Lett. 76, 1083 (1996); J. Cryst. Growth 158, 153 (1996).

[22] P. Feulner et al. (private communication).

[23] P. A. Heimann et al., J. Phys. B 20, 5005 (1987).

[24] J. Wörmer, Ph.D. thesis, University of Hamburg, Hamburg, 1990.

[25] A. Knop et al., Chem. Phys. Lett. 223, 553 (1994). 